\title{
Self-ordered cellulose nanocrystals and microscopic investigations
}

\author{
C.F. Castro-Guerrero ${ }^{1, *}$, A.B. Morales-Cepeda ${ }^{2}$, M.R. Díaz-Guillén ${ }^{3}$, \\ F. Delgado-Arroyo ${ }^{3}$, F.A. LóPEZ-GonZÁlez ${ }^{3}$ \\ ${ }^{1}$ CONACyT-Instituto Nacional de Electricidad y Energías Limpias, División de Sistemas Mecánicos, Reforma 113, Col. \\ Palmira, Cuernavaca, Mor., C.P. 62490, Mexico \\ ${ }^{2}$ Instituto Tecnológico de Ciudad Madero, División de Estudios de Posgrado e Investigación, Juventino Rosas y Jesús Urueta \\ s/n, Col. Los Mangos, Tampico, Tam., C.P. 89440, Mexico \\ ${ }^{3}$ Instituto Nacional de Electricidad y Energías Limpias, División de Sistemas Mecánicos, Reforma 113, Col. Palmira, \\ Cuernavaca, Mor., C.P. 62490, Mexico
}

\begin{abstract}
Cellulose nanocrystals were extracted from cotton. The cellulose nanocrystals made a self-assembly structure when dried under slow conditions, as it was revealed by the characterization made to the material. The AFM images of the nanocrystals showed that they had a changing local orientation, pointing in a preferred direction that underwent a periodic change. This periodic change resembles the orientation of a chiral nematic phase. The TEM images showed that the nanocrystals had a rod-like appearance with average length size of $98.5 \mathrm{~nm}$ and a diameter of $4.7 \mathrm{~nm}$. The TEM characterization showed the nanocrystals with more details than AFM. In this paper, the self-assembling of CNC was observed by AFM, and further investigations were done by TEM, deconvoluting the process of CNC nanorods aggregation.
\end{abstract}

Keywords: cellulose nanocrystals; biopolymer; self-assembly; TEM of cellulose

\section{Introduction}

Cellulose nanocrystals (CNCs) is a material that has the ability to form liquid crystals and exhibiting a self-ordering behavior [1-4]. This behavior is due to the rod-like shape of the CNCs, and has also been found in other rod-like materials [1]. The self-ordering of $\mathrm{CNC}$ arises at higher concentrations. At low concentrations, CNC suspensions are isotropic [5]. When the concentration of CNCs increases, the nanocrystals form an anisotropic phase, and this phase settles on the bottom while the isotropic phase remains on the upper part. After some time, a fingerprint pattern is formed. The chiral nematic phase shows a characteristic fingerprint pattern when observed with an optical microscope with cross-polarizers. CNCs form chiral nematic phases, but other materials have the ability to form this mesophase. Among the materials recently researched to form chiral

*E-mail: cfcastrogu@conacyt.mx nematic phase there are rod-like nanomaterials (they possess the same morphology as CNCs), such as $\mathrm{CdS}$ nanowires [6], graphene- $\mathrm{ZnO}$ nanocomposites [7], $\mathrm{ZnO}$ nanowires with graphene [8], and silicon composites with silver nanoparticles [9]. Cellulose nanocrystals are usually obtained using sulfuric acid. The acidic hydrolysis causes changes in the surface of the nanocrystals, sulfate groups are introduced in the surface of the cellulose nanocrystals, into the available $\mathrm{OH}$ groups on the surface [10]. The sulfate groups are negatively charged, and help to stabilize the cellulose nanocrystals.

CNC is extracted from cellulose, an optically active molecule. The tendency of CNC to align in a preferred direction and to form chiral nematic phases has been reported in the literature $[1,11$, 12]. We have also observed the same behavior. CNCs have been synthesized in a green way and used for reinforcing poly(vinyl alcohol) [13]. The number of papers dealing with $\mathrm{CNC}$, and its effect on the formulation of composites, have been 
increasing steadily each year [14]. The processing and purification of CNCs has been studied: in the recent years there have been several papers dealing with purification alternatives other than dialysis. One of such alternatives is neutralization using $\mathrm{NaOH}$; the results showed that it was a simple method when compared to dialysis [15]. CNCs have been used to reinforce materials, such as polymers [15], epoxy resins, wood, etc. CNCs were also used to make pickering emulsions [16], which are useful for food industry; CNCs always formed a monolayer, irrespective of the CNCs concentration, and they tended to align in the $\left[\begin{array}{lll}2 & 0 & 0\end{array}\right]$ direction. CNCs can be used as templates for fabricating chiral nematic polymers [17], taking advantage of their behavior; CNCs were used as a template for the fabrication of chiral nematic mesoporous silica microspheres. They displayed the liquid crystal behavior, formed tactoids and spherulites with a Maltese cross, characteristic of symmetrically ordered polymers. In this paper, we describe the results of our research of CNCs, showing the observation of self-ordered cellulose nanocrystals. Our results correlate to the studies described above, as the self-ordering of the CNCs is due to the ability of forming chiral nematic phases, and to the rod-like shape of the CNCs. The CNCs were observed by atomic force microscopy (AFM) after a simple drying of the sample under a saturated powder-free environment. Transmission electron microscopy (TEM) study was also performed on the cellulose nanocrystals.

\section{Experimental}

Ashless filter paper was treated with $\mathrm{NaOH}$ and $\mathrm{NaOCl}$ (commercial bleach) for removal of impurities. All the other reactants were used as received without further purification; the reactants were commercially accessible, and in the case of $\mathrm{NaOCl}$, as mentioned before, commercial bleach was used for purification. $1 \mathrm{~g}$ of purified cellulose was vigorously stirred with $17.5 \mathrm{~mL}$ of 64 wt.\% sulfuric acid at $45{ }^{\circ} \mathrm{C}$ for $45 \mathrm{~min}$. After this process, the reaction was quenched by 10 fold dilution with chilled distilled water and then the suspension was let to settle. After 2 hours, the suspension was decanted and the nanocrystals collected. The nanocrystals were again re-diluted 10 times with chilled distilled water, let to settle for 12 hours and then they were decanted again. This was done to further remove acid still on the suspension. The settled CNCs of both decantations had the same appearance. The collected nanocrystals were purified by dialysis against distilled water until the $\mathrm{pH}$ of effluent remained constant or reached neutral $\mathrm{pH}$. The dialyzed nanocrystals were sonicated with a sonicator (Sonics Vibracel VCX 750, at $65 \%$ output), for 15 minutes, divided into three periods of 5 minutes each, in order to create a colloidal suspension. The dispersed suspension of nanocrystals was filtered using Whatman glass-microfiber filters, in order to remove impurities introduced by sonication. Finally, the nanocrystals were dialyzed again in order to remove impurities introduced by the sonication. The purified CNCs were stored in a chemical refrigerator. The concentration of $\mathrm{CNC}$ suspension was measured gravimetrically by taking aliquots of $10 \mathrm{~mL}$, the weight was measured and recorded, the samples were dried and then weighed again after drying. The measured concentration was $0.45 \mathrm{wt} . \%$.

\section{Microscopic characterization}

The nanocrystals were imaged using an atomic force microscope (AFM) Nanosurf, model Naio. The images were taken in contact mode, using a silicon probe. For the AFM characterization, a freshly cleaved mica wafer was treated with a drop of polyL-lysine ( $0.1 \% \mathrm{w} / \mathrm{v}$, Sigma-Aldrich), the substrate was allowed to dry and then rinsed with distilled water, then, the substrate was saturated with drops of the CNC suspension, and it was left undisturbed to dry inside a silicon Petri dish, in a powder-free environment. The substrate was placed over a filter paper, which was saturated with the $\mathrm{CNC}$ suspension by using a Pasteur pipette; in this way the sample was let dry in a saturated environment. AFM study is usually carried out on muscovite mica wafers. One of the advantages of mica is that it is atomically flat [18]. To achieve flatness of the wafer it must be cleaved before using it. The features observed by the probe on the mica wafer are 
due to the sample or sample preparation and not due to the mica wafer, as it is atomically flat, as mentioned before. The measured thickness of the mica wafers was $313 \mu \mathrm{m}$. TEM study was performed with a Zeiss electron microscope, model Libra 120 operating at $80 \mathrm{kV} ; 10 \mu \mathrm{L}$ of the sample was deposited on a carbon-coated copper grid. After 1 minute the excess suspension was discharged, and then it was stained with a 3 wt.\% phosphotungstic acid aqueous solution [3], left for 1 minute and then the excess of PTA solution was discharged from the grid. The images were analyzed using the Gatan software without any processing or filtering. Hundred of nanocrystals were measured and their dimensions were reported.

\section{Results and discussion}

The nanocrystals showed a tendency to group and to exhibit local orientation, as shown in Fig. 1. The nanocrystal did not have a particular orientation but a local one can be observed in the image. In the upper left part of the image, the nanocrystals have the same alignment, parallel to the plane of the image. In the image, it is also observed that the CNCs have a helicoidal pattern of orientation. This pattern is caused by the tendency of the cellulose nanocrystals to form a chiral nematic phase upon reaching a specific concentration. This self-assembly of cellulose nanocrystals has been reported in CNC samples made with sulfuric acid [1, 19] and in samples having carboxylic groups on the surface: CNCs made with ammonium persulfate [3], and TEMPO-mediated CNCs [20].

The chiral nematic phase is the result of the helicoidal arrangement of cellulose nanocrystals, which arrange in layers with a preferred orientation of the cellulose nanocrystals in each layer. This behavior has been observed in other samples containing cellulose nanocrystals, and it is due to the rod-like shape of the cellulose nanocrystals. When the cellulose nanocrystals reach higher concentrations, they lack free space and so they have to order themselves. The sample observed by the AFM was taken directly from the CNC stock solution which was not diluted. During preparation, the mica wafer

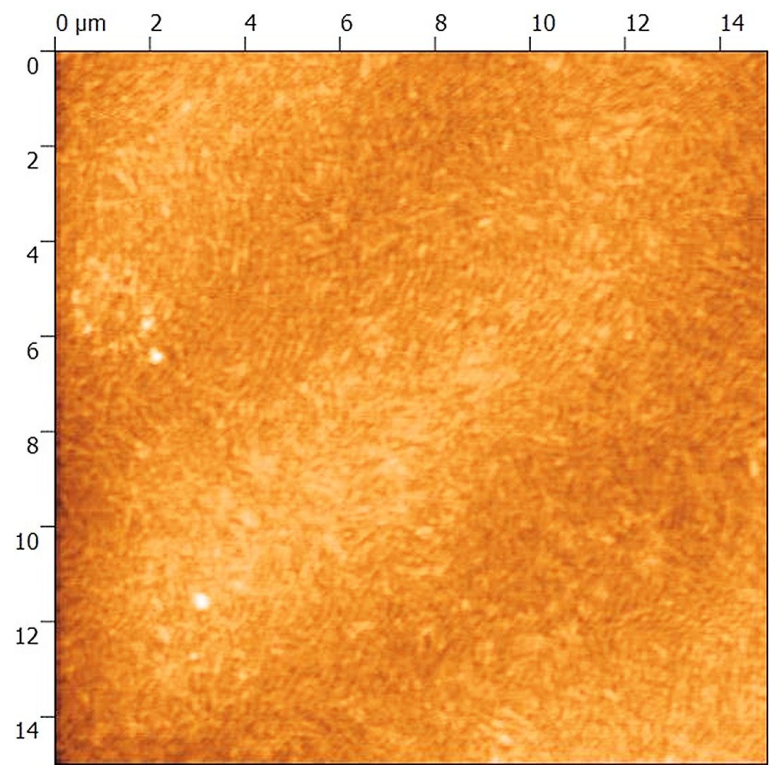

Fig. 1. AFM micrograph of cellulose nanocrystals showing self-assembly after drying. The image length is $15 \mu \mathrm{m}$. Note the general direction of the nanocrystals and the change in a twisted pattern.

was saturated with the $\mathrm{CNC}$ suspension, and the filter paper over which the wafer was placed was saturated too, as mentioned in the experimental section; no gel formation was observed during drying. The sample was dried in a closed saturated environment; therefore, the CNCs had to order during the slow drying process. During the process of drying in the saturated environment, the concentration of the particles increases, until the particles anisotropy of the colloidal solution arises and the particles are no longer able to move freely [21], and the entropy decreases. The particles have to arrange themselves in a preferred orientation; the orientation of the particles is observed in Fig. 1. When a CNC suspension is diluted, the nanocrystals tend to have no orientation, and to have a random arrangement [3]. During drying the rods have more space and freedom to have random orientation, while with higher concentration they have less space and freedom so they have to arrange in a preferred orientation. The interlayer space was estimated based on Fig. 1 by measuring the distance between two adjacent nanocrystals pointing in the same direction. This measurement corresponds to 


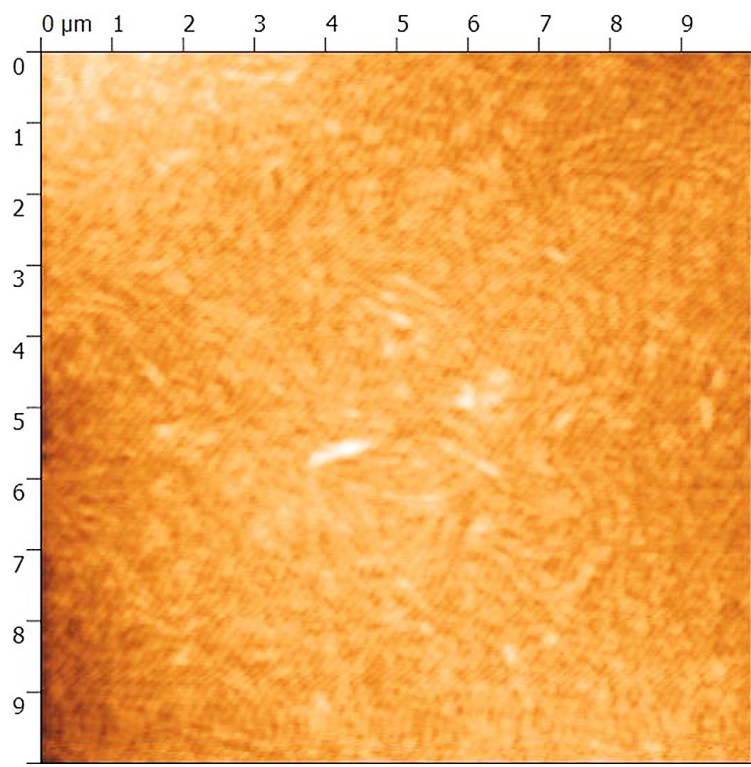

Fig. 2. AFM micrograph of cellulose nanocrystals showing self-assembly after drying at a higher magnification. The image length is $10 \mu \mathrm{m}$.

$\mathrm{P} / 2$ of the chiral nematic phase [22]. The interlayer space obtained by measuring the length between two areas of CNC with the same direction after they completed a twist, was $3.5 \mu \mathrm{m}$ [23]. The twist of the cellulosic sample might also be observed in Fig. 2, that is a higher magnification of Fig. 1. The nanocrystals forming a helical pattern, as in Fig. 1 are seen.

Fig. 3 shows the image of a diluted CNC sample. This sample was diluted, and the substrate was not saturated with drops of the CNC diluted suspension. The image shows more separated nanocrystals, with regions where there are no nanocrystals. The nanocrystals have no order in their arrangement, and they do not form a liquid crystal, nor do they have any orientation. This is the main difference compared to Fig. 1 and Fig. 2. The nanocrystals observed in the image appear as aggregated, they have a rod-like appearance and the aggregation is similar to the observed in the literature when CNC is observed by AFM.

The TEM micrograph of the nanocrystals is shown in Fig. 4. The nanocrystals have a rod-like form, similar to the observations of nanocrystals that have been reported in the literature [3]. In the

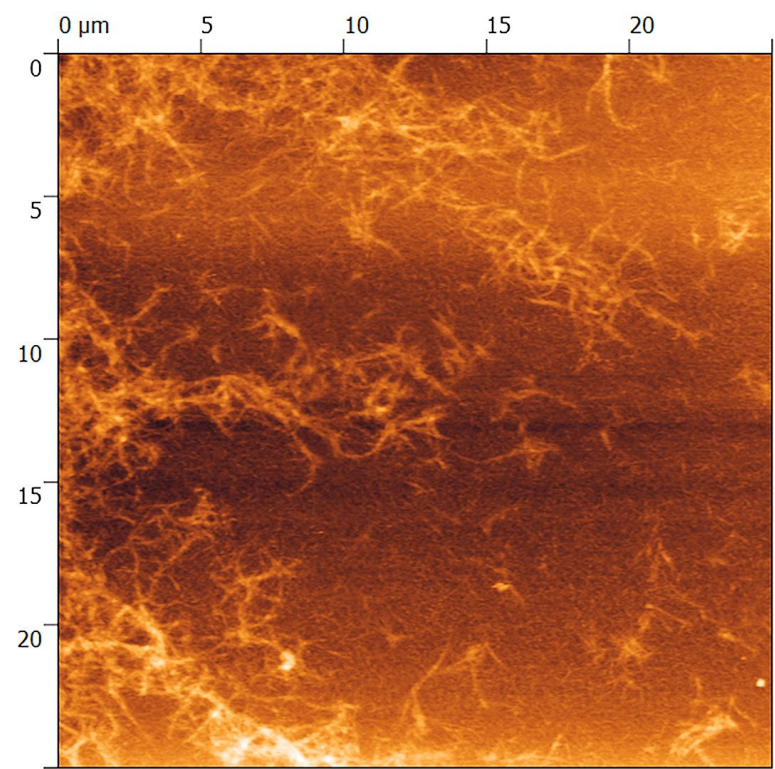

Fig. 3. AFM image of diluted cellulose nanocrystals. Size of the image is $25 \mu \mathrm{m}$.

center of the figure, there are nanocrystals that have a tendency to align in a preferred direction, as the crystals shown in the AFM images. However, they do not show the typical chiral nematic phase ordering of cellulose nanocrystals. The lack of an organized chiral nematic phase ordering may be due to the fast drying during grid preparation. The sample also shows areas of several nanocrystals very close to the areas without nanocrystals. The areas with many nanocrystals correspond to the areas in the AFM micrographs where big nanocrystals are visible, as these bigger nanocrystals correspond to aggregation of smaller nanocrystals. The aggregation of nanocrystals is mostly side-by-side, as the nanocrystals are linked together laterally, not longitudinally. The dimensions measured with TEM were the following: the length $98.5 \mathrm{~nm} \pm 5 \mathrm{~nm}$, and the diameter $4.7 \mathrm{~nm} \pm 0.2 \mathrm{~nm}$. The $\mathrm{L} / \mathrm{d}$ ratio is high. The Onsager theory predicts that high L/d ratios favor liquid crystal formation [24].

Our results are similar to the reported in the literature [25], where it was shown that cellulose nanocrystals with the length of $105 \mathrm{~nm}$ and diameter of $4.5 \mathrm{~nm}$ produced a chiral nematic pitch of $7 \mu \mathrm{m}$; in our study, we obtained cellulose nanocrystals with the length of $98.5 \mathrm{~nm}$ 
and diameter of $4.7 \mu \mathrm{m}$. The $\mathrm{P} / 2$ measured by AFM is approximately $3.5 \mu \mathrm{m}$, and the chiral nematic pitch is around $7 \mu \mathrm{m}$. Other study [3] reported that the chiral nematic pitch is $7 \mu \mathrm{m}$ whereas the length of carboxylated cellulose nanocrystals is $100 \mathrm{~nm}$ and the diameter is $6 \mathrm{~nm}$; the results suggest a relation between the length of the cellulose nanocrystals and the chiral nematic pitch. In this study and the study of Beck-Candanedo [25], the cellulose nanocrystals have sulfate groups in the surface. In other study [3], the cellulose nanocrystals have carboxylic acid on the surface. The fact that the values of the pitch reported in both studies is similar, suggests that the chiral nematic pitch is dependent on the length of the nanocrystals and independent of the functional groups on the surface. The functional groups should have an effect on the surface charge of the cellulose nanocrystals as they impart different charge to the cellulose nanocrystals.

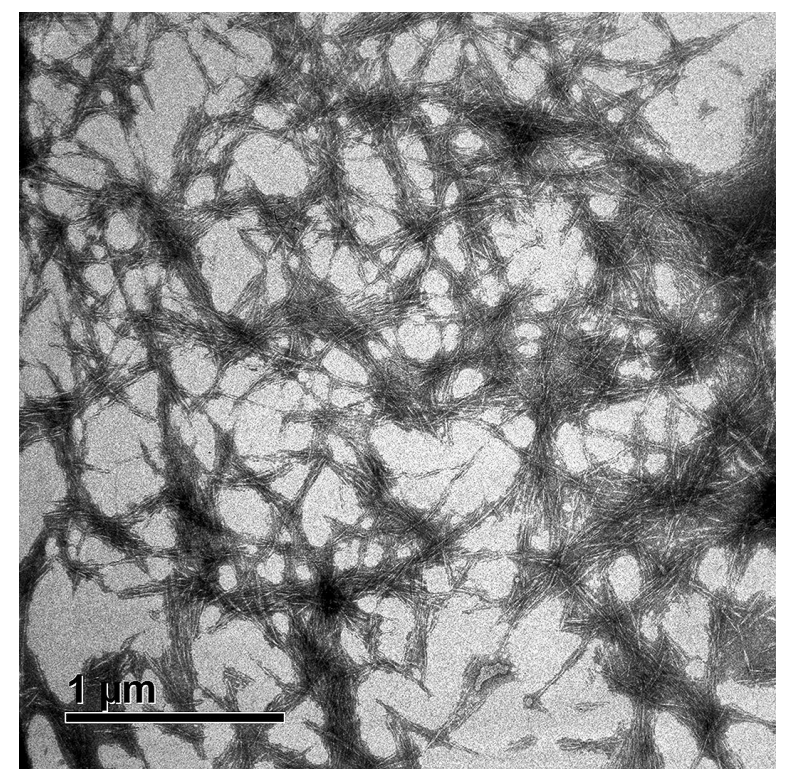

Fig. 4. TEM micrograph of the cellulose nanocrystals.

Fig. 5 shows another TEM micrograph of the CNCs. In this image, the nanocrystals are imaged with a higher magnification. The nanocrystals are observed as separated nanocrystals, with a rod-like shape. No order or orientation is observed; however, some crystals that are located close to each other point in the same direction. The orientation of the nanocrystals may be due to the intermolecular forces acting during drying of the sample, as the nanocrystals have sulfate groups on the surface. As in Fig. 4, the nanocrystals seem to be smaller than those imaged by AFM. This suggests that the images of AFM correspond to agglomerated nanocrystals. In Fig. 5, the nanocrystals are aggregated mostly laterally, as in Fig. 4, although there is also longitudinal aggregation, i.e. between the edges of the nanorods, as reported in the literature [26]. The fact that the aggregation is mostly lateral and not longitudinal, may be due to a larger contact area on the sides than on the tips, which facilitates attraction by hydrogen bonding between adjacent cellulose nanocrystals. Recent studies of CNCs using TEM have also shown lateral aggregation of the cellulose nanocrystals [27]; the aggregation occurred in freeze dried and not dried samples, and the staining method did not have impact on the aggregation behavior of the CNCs.

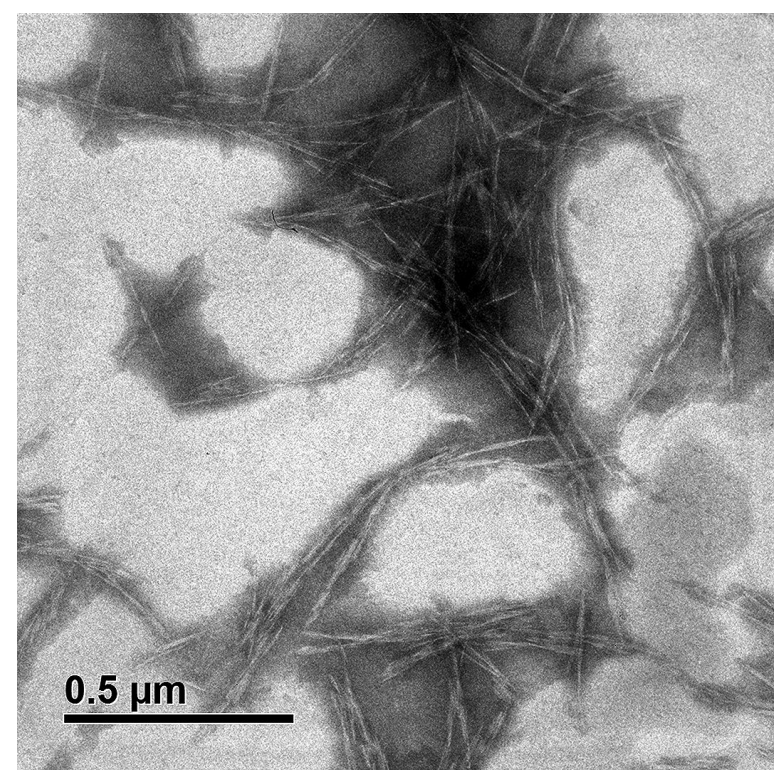

Fig. 5. TEM micrograph of the cellulose nanocrystals. The agglomeration consists of several nanocrystals.

\section{Conclusions}

Cellulose nanocrystals with helicoidal orientation have been obtained by slow drying the CNC suspensions. The nanocrystals had a tendency to self-assemble and to arrange in an ordered phase, 
as was observed in AFM images. Such behavior is characteristic of CNCs, and it is due to reduced free space of the rod-like nanocrystals when drying and reaching higher concentration [21, 24]. The CNCs had a local direction and a twist like that of the chiral nematic phase; while the diluted nanocrystals did not show the tendency to selfassembling. The TEM micrographs showed that the nanocrystals had a rod-like appearance, with narrow nanocrystals. The nanocrystals in TEM images were found to agglomerate, with those being nanoparticles in AFM images, as the nanocrystals observed by this technique have larger dimensions than the nanocrystals imaged by TEM. The agglomerated nanoparticles observed with TEM tended to group and point in a given direction.

\section{Acknowledgements}

To the CONACyT for their support. To the INEEL and the IBT-UNAM for letting us using their facilities. To Dr. Guadalupe Zavala for the TEM characterization.

\section{References}

[1] Revol J.F., Bradford H., Giasson J., MarchesSAUlt R.H., GRAY D.G., Int. J. Biol. Macromol., 14 (1992), 170.

[2] Roman M., Gray D.G., Langmuir, 21 (2005), 5555.

[3] Castro-Guerrero C.F., Gray D.G., Cellulose, 21 (2014), 2567.

[4] ZAMARripa-CERóN J.L., GARCÍA-CruZ J.C., Martínez-Arellano A.C., Castro-Guerrero C.F., Ángeles-San Martín M.E., MoralesCepeda A.B., J. Appl. Polym. Sci., 133 (2016), 43285.

[5] GRAY D.G., Nanomaterials, 6 (2016), 213.

[6] Pal K., Maiti U.N., Majumder T.P., Debnath S.C., Ghosh S., Roy S.K., Oton J.M., Nanotechnology, 24 (2013), 125702.

[7] Sagadevan S., Pal K., Chowdhury Z.Z., Foley M., J. Alloy. Compd., 728 (2017), 645.

[8] Pal K., Mohan M.L.N., Foley M., Ahmed W., Org. Electron., 56 (2018), 291.
[9] Thirugnanasambandan T., Pal K., Sidhu A., Elkodous M.A., Prasath H., KulasekarapanDiAn K., AYeshamariam A., JeEvanandam J., Nano-Struct. Nano-Objects, 16 (2018), 224.

[10] Habibi Y., Chanzy H., Vignon M.R., Cellulose, 13 (2006), 679.

[11] Roman M., Winter W.T., Biomacromolecules, 5 (2004), 1671.

[12] Habibi Y., Chanzy H., Vignon M.R., Cellulose, 13 (2006), 679.

[13] Kang X., Kuga S., Wang C., Zhao Y., Wu M., HuAnG Y., ACS Sustain. Chem. Eng., 6 (2018), 2954.

[14] Trache D., Hussin M.H., HaAFIZ M.K.H., THAKUR V.K., Nanoscale, 9 (2017), 1763.

[15] Oun A.A., Rhim J.W., Carbohydr. Polym., 134 (2015), 20.

[16] Cherhal F., Cousin F., Capron I., Biomacromolecules, 17 (2016), 496.

[17] Wang P.-X., Hamad W.Y., Maclachlan M.J., Angew. Chem. Int. Ed., 55 (2016), 1.

[18] Pillers M.A., Shute R., Farchone A., Linder K.P., Doerfler R., Gavin C., Gross V., LieberMAN., J. Vis. Exp., 101 (2015) e52972.

[19] Beck S., Bouchard J., BERry R., Biomacromolecules, 12 (2011), 167.

[20] ARAKi J., Wada M., Kuga S., Langmuir, 17 (2001), 21.

[21] Dierking I., Al-Zangana S., Nanomaterials, 7 (2017), 305.

[22] Gray D.G., Mu X., Materials, 8 (2015), 7873.

[23] Parker R.M., Frka-Petesic B., Guidetti G., Kamita G., Consani G., Abell C., Vignolini S., ACS Nano, 10 (2016), 8443.

[24] Onsager L., Ann. N. Y. Acad. Sci., 51 (1949), 627.

[25] Beck-Candanedo S., Roman M., Gray D.G., Biomacromolecules, 6 (2005), 1048.

[26] Honorato-Ríos C., Lehr C., SAnCtuary R., OsIPOV M.A., BALLER J., LAGERWALL J.P.F., NPG Asia Mater, 10 (2018), 455.

[27] Stinson-Bagby K.L., Roberts R., Foster J., Carbohydr. Polym., 186 (2018), 429. 